\title{
ENHANCING PD-CONTROLLER EFFICIENCY VIA TIME- DELAYS TO SUPPRESS NONLINEAR SYSTEM OSCILLATIONS
}

\section{W. A. EL-Ganaini}

Department of Physics and Engineering Mathematics, Faculty of Electronic Engineering, Menouf, 32952, Menoufia University, Egy pt. *Corresponding author contact details (e-mail address): wedad.ali.el.ganaini@gmail.com

Received 23/5/2017

Revised 28/6/2017

Accepted 12/7/2017

Within this work, the conventional PD-controller is proposed to suppress the lateral oscillations of a nonlinear Jeffcott-rotor system. Time-delays in the control loop are included in the system model. The slow-flow modulating equations governing the whole system dynamics are obtained. Influence of the controller gains and time-delays on response curves are explored. The analyses showed that the controller efficiency has been enhanced at specific values of time-delays; while the system may lose its stability at the other values. The phenomenon of enhancing the controller efficiency was explained and accordingly the optimal values of the loopdelays have been reported.

Keywords Time-delays control, Limit point, Hopf-bifurcations, Whirling-orbit, Multiple scales method Nomenclature

$$
\begin{gathered}
x_{1}, \dot{x}_{1}, \ddot{x}_{1} \\
x_{2}, \dot{x}_{2}, \ddot{x}_{2} \\
\mu_{1}, \mu_{2} \\
\alpha \\
\delta_{1}, \delta_{2} \\
\tau_{1}, \tau_{2} \\
\Omega \\
f \\
a_{1}, a_{2} \\
\sigma
\end{gathered}
$$$$
\text { Displacement, velocity and acceleration at } X_{1} \text { direction. }
$$$$
\text { Displacement, velocity and acceleration at } X_{2} \text { direction. }
$$$$
\text { Linear damping coefficients of both vibration modes. }
$$$$
\text { Cubic nonlinearity coefficient. }
$$$$
\text { The position and velocity gains of the position-velocity controller, respectively. }
$$$$
\text { Time-delays in the control loop. }
$$$$
\text { The disc spinning speed. }
$$$$
\text { The disc eccentricity. }
$$$$
\text { Oscillation amplitudes at } X_{1} \text { and } X_{2} \text { directions, respectively. }
$$

External detuning parameter that characterizes the closeness of the disc spinning speed $\Omega$ to the normalized critical speed where $\Omega=1+\sigma$.

\section{Mathematics Subject Classification 70K99, 34E13, and 34D10}

\section{Introduction}

The dynamical interaction among the stator, rotating parts and the mass imbalance of the rotor are the main reasons of the rotating machinery vibrations. Due to the wide industrial applications of the rotating machines, hundreds of research articles are dedicated annually to study and control the nonlinear oscillations of those machines. Generally, the vibration control methods are classified into three main categories, which are active, semi-active, and passive control methods. Involving active controllers in the control loop raises the wellknown problem of time-delays that may be the cause of controlled system instability. Many of the recently published papers concerning vibration control have discussed the influences of time-delays on the controlled systems stability and the connected controller performance, where Hu et al. [1] introduced time-delayed state feedback controller to suppress the nonlinear oscillations of Duffing oscillator at primary and sub-harmonic resonance cases. The analyses illustrated that involving time-delays in the control loop can provide active damping resulting in the enhancement of the controller efficiency. Moreover, the authors concluded that the combination of positive position and negative velocity feedback control could mitigate the resonant peak efficiently. Maccari studied the nonlinear vibrations control of van der pol oscillator when excited parametrically 
[2] and externally [3], utilizing time-delayed state feedback controller. He concluded that the feedback gains and time-delays could reduce the vibration peaks and suppress the system quasi-periodic motions at both external and parametric excitations. Maccari [4] discussed the nonlinear vibrations of a harmonically excited cantilever beam model at the primary resonance case. Time-delayed position-velocity controller was employed to suppress the system oscillations, where the author concluded that the appropriate choice of the controller gains and loop-delays might enhance the controller efficiency. Maccari discussed the vibration control of van der pol oscillator and a parametrically excited nonlinear system by integrating time-delayed state feedback controller $[5,6]$. The author constructed what he defined by "stability chart" for the amplitude and phase of the periodic phase-locked solutions. Then he showed that it is possible to calculate the feedback gains and time-delays required to generate specific vibration amplitude. Morrison et al. [7] investigated the nonlinear Mathieu equation at 2:1 parametric resonance and time-delayed positive-position feedback controller. They concluded that involving time-delay into an undamped parametrically excited system increases the linear damping of the system. Time-delayed positionvelocity controller was utilized to suppress the free vibrations of van der Pol oscillator [8], where the authors applied the two variable expansion method to obtain two Hopf curves that are plotted in control-gain time-delay plane. They showed that the obtained Hopf-curves include a region at which the system vibrations are eliminated and the oscillation amplitude could be suppressed by appropriately setting the loop-delay within this region. Shao et al. [9] investigated the effects of time-delays on dynamics of a nonlinear Microelectromechanical system. Timedelayed position-velocity feedback controller is applied to suppress the system vibrations. The authors concluded that the existence of time-delays in the case of negative feedback destabilize the system motions, while the timedelays enhance the controller efficiency at positive-position and negative-velocity feedback control. The nonlinear vibrations of a nonlinear system having quadratic, cubic, quartic, and quantic nonlinearity has been discussed in Ref. [10]. Time-delayed position-velocity feedback controller is employed to suppress the system vibrations. The authors reported that suitable choice of the time-delays and feedback gains reduces the system resonant peaks. Different time-delayed control algorithms to suppress the nonlinear vibrations of a cantilever beam model when excited externally and parametrically have been studied in Refs [11-13]. In Ref. [14], Xu et al. introduced Parameter estimation technique for the second order systems from the step responses based on the Newton iteration method, while in [15] they proposed a tuning method for the PD-controller parameters to control first-order time-delayed system. Saeed and El-Ganaini [16, 17] applied time-delayed position-velocity feedback controller to mitigate the nonlinear oscillations of a horizontally supported nonlinear Jeffcott-rotor systems. They illustrated simple and concrete method to calculate the optimal values of time-delays at both the negative and positive feedback control. Then, they showed that involving time-delays in the control loop could enhance the controller effectiveness from $80 \%$ to $95 \%$.

In this work, the lateral vibration of the vertically supported nonlinear Jeffcott-rotor system [16] is studied. The position-velocity (PD) feedback controller is proposed to control the considered system vibrations. The time-delays $\left(\tau_{1}\right.$ and $\left.\tau_{2}\right)$ in the control loop are included in the system model. Sensitivity of the system response curves to the controller gains and loop-delays are explored. Then, stability chart of the controlled system is illustrated to show the stable and unstable solutions regions in $\tau_{1} \tau_{2}$-plane. The effects of increasing both the position gain $\left(\delta_{1}\right)$ and velocity gain $\left(\delta_{2}\right)$ on the stability chart topology are illustrated. The analysis revealed the possibility of selecting time-delays values in some way to improve the controller efficiency in suppressing the system vibrations. The reported optimal values of time-delays are illustrated analytically and were confirmed numerically. Then, we derived too simple algebraic equations that can be used to calculate the optimal control parameters (i.e. $\delta_{1}, \delta_{2}, \tau_{1}$, and $\tau_{2}$ ) for the best control performance.

\section{Mathematical model and asymptotic analyses}

The nonlinear differential equations governing the lateral vibrations in $X_{1}$ and $X_{2}$ directions of a vertically supported Jeffcott-rotor system shown in Fig. 1 are given as follow:

$$
\begin{aligned}
& m \ddot{x}_{1}+c_{x} \dot{x}_{1}+R_{x_{1}}=m e_{d} v^{2} \cos (v t) \\
& m \ddot{x}_{2}+c_{y} \dot{x}_{2}+R_{x_{2}}=m e_{d} v^{2} \sin (v t)
\end{aligned}
$$


where, $m$ is the disc mass, $c_{x}, c_{y}$ are the linear damping coefficients at $X_{1}$ and $X_{2}$ directions, $R_{x_{1}}, R_{x_{2}}$ are the nonlinear restoring forces at $X_{1}$ and $X_{2}$ directions, $e_{d}$ denotes the disc eccentricity, $v$ is the disc spinning speed, and $t$ is the time. The restoring force are considered as a nonlinear function of the distance $r$ of the rotating disc from the geometric center $G$ as shown in Fig. 1b as [18]:

$$
R(r)=k_{1} r+k_{2} r^{3}, \quad r=\sqrt{x_{1}^{2}+x_{2}^{2}}
$$

Accordingly, the restoring forces in $X_{1}$ and $X_{2}$ directions are given as:

$$
\begin{aligned}
& R_{x_{1}}=\left(k_{1} r+k_{2} r^{3}\right) \cos (\theta)=\left(k_{1}+k_{2} r^{2}\right) r \cos (\theta)=k_{1} x_{1}+k_{2}\left(x_{1}^{3}+x_{1} x_{2}^{2}\right) \\
& R_{x_{2}}=\left(k_{1} r+k_{2} r^{3}\right) \sin (\theta)=\left(k_{1}+k_{2} r^{2}\right) r \sin (\theta)=k_{1} x_{2}+k_{2}\left(x_{1}^{2} x_{2}+x_{2}^{3}\right)
\end{aligned}
$$

Substituting equations (3.1) and (3.2) into equations (1.1) and (1.2), we get

$$
\begin{aligned}
& m \ddot{x}_{1}+c_{x} \dot{x}_{1}+k_{1} x_{1}+k_{2} x_{1}^{3}+k_{2} x_{1} x_{2}^{2}=m e_{d} v^{2} \cos (v t) \\
& m \ddot{x}_{2}+c_{y} \dot{x}_{2}+k_{1} x_{2}+k_{2} x_{1}^{2} x_{2}+k_{2} x_{2}^{3}=m e_{d} v^{2} \sin (v t)
\end{aligned}
$$

By introducing the dimensionless parameters $t^{*}=\omega t, \omega=\sqrt{k_{1} / m}, x_{1}^{*}=x_{1} / e, x_{2}^{*}=x_{2} / e$ into equations (4.1) and (4.2), and omitting the asterisks for brevity, we get

$\ddot{x}_{1}+\mu_{1} \dot{x}_{1}+x_{1}+\alpha x_{1}^{3}+\alpha x_{1} x_{2}^{2}=f \Omega^{2} \cos (\Omega t)$

$\ddot{x}_{2}+\mu_{2} \dot{x}_{2}+x_{2}+\alpha x_{1}^{2} x_{2}+\alpha x_{2}^{3}=f \Omega^{2} \sin (\Omega t)$

where $\mu_{1}=\frac{c_{x}}{\sqrt{m k_{1}}}, \mu_{2}=\frac{c_{y}}{\sqrt{m k_{1}}}, \alpha=\frac{e^{2} k_{2}}{k_{1}}, f=\frac{e_{d}}{e}, \Omega=\frac{v}{\omega}$. By integrating a time-delayed conventional PDcontroller to the system equations (5.1) and (5.2) as shown in Fig. 1c, the governing equations of the whole system become as follows:

$$
\begin{aligned}
& \ddot{x}_{1}+\mu_{1} \dot{x}_{1}+x_{1}+\alpha x_{1}^{3}+\alpha x_{1} x_{2}^{2}=f \Omega^{2} \cos (\Omega t)-\left(\delta_{1} x_{1}\left(\mathrm{t}-\tau_{1}\right)+\delta_{2} \dot{x}_{1}\left(\mathrm{t}-\tau_{2}\right)\right) \\
& \ddot{x}_{2}+\mu_{2} \dot{x}_{2}+x_{2}+\alpha x_{2}^{3}+\alpha x_{1}^{2} x_{2}=f \Omega^{2} \sin (\Omega t)-\left(\delta_{1} x_{2}\left(\mathrm{t}-\tau_{1}\right)+\delta_{2} \dot{x}_{2}\left(\mathrm{t}-\tau_{2}\right)\right)
\end{aligned}
$$

where $\delta_{1} x_{1}\left(t-\tau_{1}\right)+\delta_{2} \dot{x}_{1}\left(t-\tau_{2}\right)$ and $\delta_{1} x_{2}\left(t-\tau_{1}\right)+\delta_{2} \dot{x}_{2}\left(t-\tau_{2}\right)$ represent the control forces at $X_{1}$ and $X_{2}$ directions, respectively.

(a)

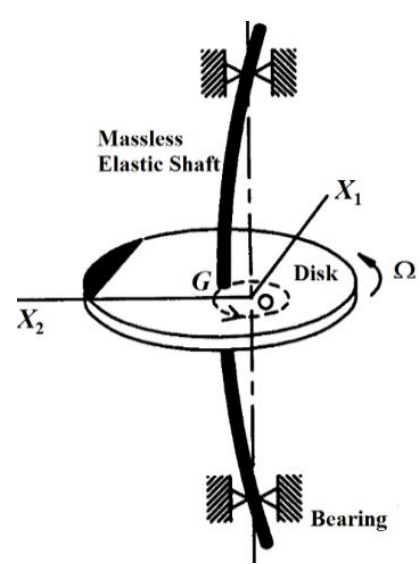

(b)

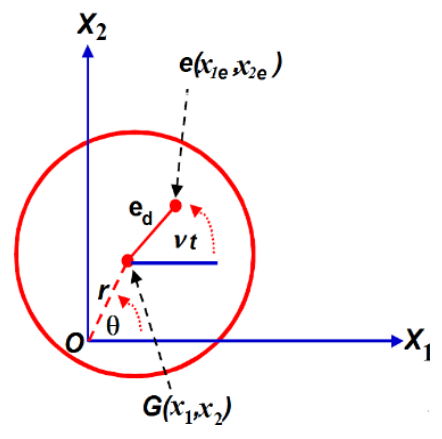

(c)

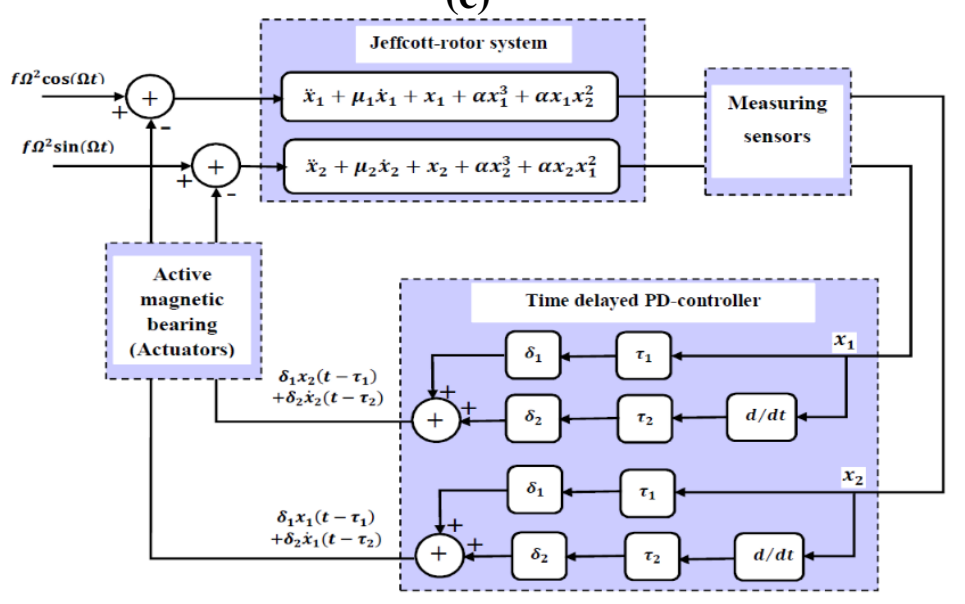

Fig. $1(\mathbf{a}, \mathbf{b})$ Jeffcott-rotor system, and (c) schematic diagram of the controlled Jeffcott-rotor system

\subsection{Asymptotic analysis and slow-flow governing equations}

In this section, the multiple scales perturbation technique [19] is applied seeking an approximate solution to equations (6.1) and (6.2) as follows:

$$
x_{k}(t, \varepsilon)=x_{k 1}\left(T_{0}, T_{1}\right)+\varepsilon x_{k 2}\left(T_{0}, T_{1}\right)+O\left(\varepsilon^{2}\right), \quad k=1,2
$$


$x_{k}\left(t-\tau_{j}, \varepsilon\right)=x_{k 1 \tau_{j}}\left(T_{0}, T_{1}\right)+\varepsilon x_{k 2 \tau_{j}}\left(T_{0}, T_{1}\right)+O\left(\varepsilon^{2}\right), \quad k=1,2, \quad j=1,2$

where $\varepsilon$ is a small dimensionless perturbation parameter used as a book-keeping parameter, $T_{j}=\varepsilon^{j} t, j=0,1$ are two time scales that describe the fast and slow scales of the system motions. The time derivatives in equations (6.1) and (6.2) can be expressed in terms of $T_{0}$ and $T_{1}$ as:

$\frac{d}{d t}=D_{0}+\varepsilon D_{1} \quad \frac{d^{2}}{d t^{2}}=D_{0}^{2}+2 \varepsilon D_{0} D_{1}, \quad D_{j}=\frac{\partial}{\partial T_{j}}, \quad j=0,1$

The system parameters are scaled according to their values as follows:

$\mu_{j}=\varepsilon \hat{\mu}_{j}, \alpha=\varepsilon \hat{\alpha}, \quad \delta_{1}=\varepsilon \hat{\delta}_{1}, \quad \delta_{2}=\varepsilon \hat{\delta}_{2}, \quad f=\varepsilon \hat{f}: \quad j=1,2$.

Substituting equations (7.1), (7.2), (8), and (9) into equations (6.1) and (6.2), with equating coefficients of like powers of $\varepsilon$, we have

$O\left(\varepsilon^{0}\right)$ :

$\left(D_{0}^{2}+1\right) x_{11}=0$

$\left(D_{0}^{2}+1\right) x_{21}=0$

$O\left(\varepsilon^{1}\right)$ :

$\left(D_{0}^{2}+1\right) x_{12}=-2 D_{0} D_{1} x_{11}-\hat{\mu}_{1} D_{0} x_{11}-\hat{\alpha} x_{11}^{3}-\hat{\alpha} x_{11} x_{21}^{2}+\hat{f} \Omega^{2} \cos \left(\Omega T_{0}\right)-\left(\hat{\delta} x_{11 \tau_{1}}+\hat{\delta}_{2} D_{0} x_{11 \tau_{2}}\right)$

$\left(D_{0}^{2}+1\right) x_{22}=-2 D_{0} D_{1} x_{21}-\hat{\mu}_{2} D_{0} x_{21}-\hat{\alpha} x_{21}^{3}-\hat{\alpha} x_{11}^{2} x_{21}+\hat{f} \Omega^{2} \sin \left(\Omega T_{0}\right)-\left(\hat{\delta}_{1} x_{21 \tau_{1}}+\hat{\delta}_{2} D_{0} x_{21 \tau_{2}}\right)$

The solution of equations (10.1) and (10.2), can be expressed as

$x_{11}\left(T_{0}, T_{1}\right)=Q_{1}\left(T_{1}\right) e^{i T_{0}}+\bar{Q}_{1}\left(T_{1}\right) e^{-i T_{0}}$

$x_{21}\left(T_{0}, T_{1}\right)=Q_{2}\left(T_{1}\right) e^{i T_{0}}+\bar{Q}_{2}\left(T_{1}\right) e^{-i T_{0}}$

where, the coefficients $Q_{1}\left(T_{1}\right)$ and $Q_{2}\left(T_{1}\right)$ are unknown functions of $T_{0}$ and $T_{1}$. Substituting equations (12.1) and (12.2) into equations (11.1) and (11.2), yields

$$
\begin{aligned}
\left(D_{0}^{2}+1\right) x_{12}= & -2 i D_{1} Q_{1} e^{i T_{0}}-\hat{\mu}_{1} i Q_{1} e^{i T_{0}}-\hat{\alpha} Q_{1}^{3} e^{3 i T_{0}}-3 \hat{\alpha} Q_{1}^{2} \bar{Q}_{1} e^{i T_{0}}-\hat{\alpha} Q_{1} Q_{2}^{2} e^{3 i T_{0}}-\hat{\alpha} \bar{Q}_{1} Q_{2}^{2} e^{i T_{0}} \\
& -2 \hat{\alpha} Q_{1} Q_{2} \bar{Q}_{2} e^{i T_{0}}-\hat{\delta} i Q_{1} e^{i\left(T_{0}-\tau_{2}\right)}-\hat{\delta}_{1} Q_{1} e^{i\left(T_{0}-\tau_{1}\right)}+\frac{\hat{f} \Omega^{2}}{2} e^{i \Omega T_{0}}+c c \\
\left(D_{0}^{2}+1\right) x_{22}= & -2 i D_{1} Q_{2} e^{i T_{0}}-\hat{\mu}_{2} i Q_{2} e^{i T_{0}}-\hat{\alpha} Q_{2}^{3} e^{3 i T_{0}}-3 \hat{\alpha} Q_{2}^{2} \bar{Q}_{2} e^{i T_{0}}-\hat{\alpha} Q_{1}^{2} Q_{2} e^{3 i T_{0}}-\hat{\alpha} Q_{1}^{2} \bar{Q}_{2} e^{i T_{0}} \\
& -2 \hat{\alpha} Q_{1} \bar{Q}_{1} Q_{2} e^{i T_{0}}-\hat{\delta}_{2} i Q_{2} e^{i\left(T_{0}-\tau_{2}\right)}-\hat{\delta}_{1} Q_{2} e^{i\left(T_{0}-\tau_{1}\right)}-i \frac{\hat{f} \Omega^{2}}{2} e^{i \Omega T_{0}}+\mathrm{cc}
\end{aligned}
$$

Before we proceed further, the possible resonance cases must be determine at this approximation order, which is primary resonance (i.e. $\Omega \cong 1$ ). So, the closeness of the considered resonance case can be described by introducing the detuning parameters $\sigma$ according to

$\Omega=1+\sigma=1+\varepsilon \hat{\sigma}$

Inserting equation (14) into the small-divisor and secular terms of equations (13.1) and (13.2), yields the following solvability conditions

$2 i D_{1} Q_{1}+\hat{\mu}_{1} i Q_{1}+3 \hat{\alpha} Q_{1}^{2} \bar{Q}_{1}+\hat{\alpha} \bar{Q}_{1} Q_{2}^{2}+2 \hat{\alpha} Q_{1} Q_{2} \bar{Q}_{2}+\hat{\delta}_{2} i Q_{1} e^{-i \tau_{2}}+\hat{\delta}_{1} Q_{1} e^{-i \tau_{1}}-\frac{\hat{f} \Omega^{2}}{2} e^{i \hat{\sigma} T_{1}}=0$
$2 i D_{1} Q_{2}+\hat{\mu}_{2} i Q_{2}+3 \hat{\alpha} Q_{2}^{2} \bar{Q}_{2}+\hat{\alpha} Q_{1}^{2} \bar{Q}_{2}+2 \hat{\alpha} Q_{1} \bar{Q}_{1} Q_{2}+\hat{\delta_{2}} i Q_{2} e^{-i \tau_{2}}+\hat{\delta_{1}} Q_{2} e^{-i \tau_{1}}+i \frac{\hat{f} \Omega^{2}}{2} e^{i \hat{\sigma} T_{1}}=0$

To analyze equations (15.1) and (15.2), we can express the unknown functions $Q_{1}\left(T_{1}\right)$ and $Q_{2}\left(T_{1}\right)$ in polar form as: 
$Q_{j}=\frac{1}{2} a_{j} e^{i \psi_{j}} \Rightarrow D_{1} Q_{j}=\frac{d}{d T_{1}} Q_{j}=\frac{d}{\varepsilon d t} Q_{j}=\frac{1}{\varepsilon} \dot{Q}_{j}=\frac{1}{2 \varepsilon}\left(\dot{a}_{j} e^{i \psi_{j}}+i a_{j} \dot{\psi}_{j} e^{i \psi_{j}}\right), \quad j=1,2$

Substiting equations (16) into equations (15.1) and (15.2) with returning each scaled parameter to its original form (i.e. $\hat{\mu}_{1}=\frac{\mu_{1}}{\varepsilon}, \hat{\mu}_{2}=\frac{\mu_{2}}{\varepsilon}, \hat{\alpha}=\frac{\alpha}{\varepsilon}, \hat{\delta}_{1}=\frac{\delta_{1}}{\varepsilon}, \hat{\delta}_{2}=\frac{\delta_{2}}{\varepsilon}, \hat{f}=\frac{f}{\varepsilon}, \hat{\sigma}=\frac{\sigma}{\varepsilon}, T_{1}=\varepsilon t$ ), we get the following slowflow modulating equations governing the modulation-amplitudes and the modified phases of the system.

$$
\begin{aligned}
& \dot{a}_{1}=-\frac{1}{2}\left\{\underline{\underline{\mu_{1}-\delta_{1} \sin \left(\tau_{1}\right)+\delta_{2} \cos \left(\tau_{2}\right)}}\right\} a_{1}+\frac{\alpha}{8} a_{1} a_{2}^{2} \sin \left(2 \theta_{1}-2 \theta_{2}\right)-\frac{f \Omega^{2}}{2} \sin \left(\theta_{1}\right) \\
& \dot{a}_{2}=-\frac{1}{2}\left\{\underline{\underline{\mu_{2}-\delta_{1} \sin \left(\tau_{1}\right)+\delta_{2} \cos \left(\tau_{2}\right)}}\right\} a_{2}-\frac{\alpha}{8} a_{1}^{2} a_{2} \sin \left(2 \theta_{1}-2 \theta_{2}\right)-\frac{f \Omega^{2}}{2} \cos \left(\theta_{2}\right) \\
& \dot{\theta}_{1}=-\left\{\underline{\underline{\sigma-\frac{1}{2}}\left(\delta_{1} \cos \left(\tau_{1}\right)+\delta_{2} \sin \left(\tau_{2}\right)\right)}\right\}+\frac{3 \alpha}{8} a_{1}^{2}+\frac{\alpha}{4} a_{2}^{2}+\frac{\alpha}{8} a_{2}^{2} \cos \left(2 \theta_{1}-2 \theta_{2}\right)-\frac{f \Omega^{2}}{2 a_{1}} \cos \left(\theta_{1}\right) \\
& \dot{\theta}_{2}=-\left\{\underline{\underline{\sigma-\frac{1}{2}\left(\delta_{1} \cos \left(\tau_{1}\right)+\delta_{2} \sin \left(\tau_{2}\right)\right)}}\right\}+\frac{3 \alpha}{8} a_{2}^{2}+\frac{\alpha}{4} a_{1}^{2}+\frac{\alpha}{8} a_{1}^{2} \cos \left(2 \theta_{1}-2 \theta_{2}\right)+\frac{f \Omega^{2}}{2 a_{2}} \sin \left(\theta_{2}\right)
\end{aligned}
$$

where $\theta_{1}=\psi_{1}-\sigma t, \theta_{2}=\psi_{2}-\sigma t$. By examining the first terms of equations (17.1) to (17.4), it is noted that the integration of the time-delayed position-velocity feedback controller to the system has modified its linear damping coefficients $\mu_{1}, \mu_{2}$ to the equivalent coefficients $\mu_{e 1}=\mu_{1}-\delta_{1} \sin \left(\tau_{1}\right)+\delta_{2} \cos \left(\tau_{2}\right)$ and $\mu_{e 2}=\mu_{2}-$ $\delta_{1} \sin \left(\tau_{1}\right)+\delta_{2} \cos \left(\tau_{2}\right)$, while the detuning parameter $\sigma$ is modified to $\sigma_{e}=\sigma-\frac{1}{2}\left[\delta_{1} \cos \left(\tau_{1}\right)+\delta_{2} \sin \left(\tau_{2}\right)\right]$. Accordingly, it is possible to select the loop-delays ( $\tau_{1}$ and $\tau_{2}$ ) in some way to maximize $\mu_{e 1}$ and $\mu_{e 2}$ and hence enhancing the controller efficiency.

\subsection{Steady-state lateral oscillations and stability investigations}

Setting $\dot{a}_{1}=\dot{a}_{2}=\dot{\theta}_{1}=\dot{\theta}_{2}=0$ into equations (17.1) to (17.4), we get four nonlinear algebraic equations. These equations describe the steady state whirling motions of the considered system in terms of the controller parameters $\left(\delta_{1}, \delta_{2}, \tau_{1}, \tau_{2}\right)$. Let $\left(a_{10}, a_{20}, \theta_{10}, \theta_{20}\right)$ is the steady state solution of these algebraic equations. To study the stability of that solution $\left(a_{10}, a_{20}, \theta_{10}, \theta_{20}\right)$, we examine the solution behaviors under small perturbations $a_{11}, a_{21}, \theta_{11}$, and $\theta_{21}$. Therefore, we assume

$a_{1}=a_{11}+a_{10}, a_{2}=a_{21}+a_{20}, \theta_{1}=\theta_{11}+\theta_{10}, \theta_{2}=\theta_{21}+\theta_{20} \Rightarrow \dot{a}_{1}=\dot{a}_{11}, \dot{a}_{2}=\dot{a}_{21}, \dot{\theta}_{1}=\dot{\theta}_{11}, \dot{\theta}_{2}=\dot{\theta}_{21}$

Substituting equation (18) into equations (17.1) to (17.4), and expanding for small $a_{11}, a_{21}, \theta_{11}$, and $\theta_{21}$, keeping the linear terms only (i.e. linearization of nonlinear system (17.1)-(17.4), around the equilibrium point $\left.\left(a_{10}, a_{20}, \theta_{10}, \theta_{20}\right)\right)$, we have

$\left(\begin{array}{l}\dot{a}_{11} \\ \dot{\theta}_{11} \\ \dot{a}_{21} \\ \dot{\theta}_{21}\end{array}\right)=\left(\begin{array}{llll}\beta_{11} & \beta_{12} & \beta_{13} & \beta_{14} \\ \beta_{21} & \beta_{22} & \beta_{23} & \beta_{24} \\ \beta_{31} & \beta_{32} & \beta_{33} & \beta_{34} \\ \beta_{41} & \beta_{42} & \beta_{43} & \beta_{44}\end{array}\right)\left(\begin{array}{c}a_{11} \\ \theta_{11} \\ a_{21} \\ \theta_{21}\end{array}\right)$

The above square matrix is the system Jacobian matrix. Thus, the stability of the steady state oscillations depends on the eigenvalues of the above matrix. Accordingly, one can obtain the following eigenvalue equations.

$\begin{array}{llll}\beta_{11}-\lambda & \beta_{12} & \beta_{13} & \beta_{14} \\ \beta_{21} & \beta_{22}-\lambda & \beta_{23} & \beta_{24} \\ \beta_{31} & \beta_{32} & \beta_{33}-\lambda & \beta_{34} \\ \beta_{41} & \beta_{42} & \beta_{43} & \beta_{44}-\lambda\end{array} \mid=0$

Expanding the determinant (20), yields

$\lambda^{4}+\rho_{1} \lambda^{3}+\rho_{2} \lambda^{2}+\rho_{3} \lambda+\rho_{4}=0$ 
where $\lambda$ denotes the eigenvalues of the Jacobian matrix, and $\rho_{1}, \rho_{2}, \rho_{3}$ and $\rho_{4}$ are the coefficient of equation (21). Applying Routh-Hurwitz stability criterion, the necessary and sufficient conditions for the solution $\left(a_{10}\right.$, $\left.a_{20}, \theta_{10}, \theta_{20}\right)$ to be stable are

$\rho_{1}>0, \rho_{1} \rho_{2}-\rho_{3}>0, \rho_{3}\left(\rho_{1} \rho_{2}-\rho_{3}\right)-\rho_{1}^{2} \rho_{4}>0, \quad \rho_{4}>0$

\section{Results and discussions}

In this section, the steady state lateral vibration of a nonlinear vertically suspended Jeffcott-rotor system before and after control are investigated via solving the nonlinear algebraic equations (17.1) to (17.4) when $\dot{a}_{1}=\dot{a}_{2}=$ $\dot{\theta}_{1}=\dot{\theta}_{2}=0$. Newton-Raphson algorithm is employed to solve these equations at the system parameters: $f=$ 0.025, $\alpha=0.05, \mu_{1}=\mu_{2}=0.015, \sigma_{1}=0.0, \Omega=1+\sigma, \delta_{1}=0.1, \delta_{2}=0.02, \tau_{1}=\tau_{2}=0.0, \quad$ unless otherwise is mentioned. The response curves are obtained by varying the detuning parameter $(\sigma)$ or the disc eccentricity $(f)$ as a bifurcation control parameter and plotting the corresponding vibration amplitudes $\left(a_{1}, a_{2}\right)$. In addition, the obtained solution stability is reported via checking the conditions (22). Then, the obtained solution is plotted as a solid line if it is stable and as a dashed one if it is unstable as shown in Figs 2-3, 9-11, 14. Moreover, to confirm the accuracy of the obtained bifurcation diagrams, the non-autonomous system equations (6.1) and (6.2) have been solved numerically using the Matlab solvers DDE23 [20] (when $\tau_{1}, \tau_{2}>0.0$ ) and DDE45 (when $\tau_{1}=\tau_{2}=0.0$ ), then the steady state vibration amplitudes that obtained numerically are illustrated on the bifurcation diagrams as big-dots as shown in Figs 3, 4, 10, and 13. Frequency Response Curve (FRC) of the Jeffcott rotor system before control (i.e. $\delta_{1}=\delta_{2}=0.0$ ) is displayed in Fig. 2 at different levels of the disc eccentricity $(f)$. The figure shows that the system responds as a semi-linear system as long as $f \leq 0.01$, while if $f>0.01$, the nonlinearity dominates the system response and the bistable vibrating amplitudes appear at specific range of the disc spinning speed. Accordingly, the nonlinear Jeffcott rotor system can execute one of two whirling orbits depending on the initial position of the rotating disc. Fig. 3 compares the system FRC before control and after applying negative-position and negative-velocity feedback controller at zero time-delays (i.e. $\delta_{1}=0.1, \delta_{2}=0.02$, and $\tau_{1}=\tau_{2}=0.0$ ). The figure illustrates that the connection of the proposed controller to the system has eliminated the bistable solutions interval (i.e. eliminates jump phenomenon) and reduced the vibration peaks to approximately one half. Fig. 4 illustrates the system FRC before and after applying positiveposition and negative-velocity feedback controller (i.e. $\delta_{1}=-0.1, \delta_{2}=0.02$, and $\tau_{1}=\tau_{2}=0.0$ ). It is noticed from Figs. 3 and 4 that the positive-position negative-velocity feedback controller has high efficiency in mitigating the system vibrations than the negative-position negative-velocity controller. Stability of the controlled system as a function of the time-delays is illustrated in $\tau_{1} \tau_{2}$-plane in Fig. 5, where Fig. 5a shows the stability chart at negative-position negative-velocity feedback control (i.e. $\delta_{1}=0.1, \delta_{2}=0.02$ ), while Fig. $5 \mathrm{~b}$ displays the stability chart at positive-position negative-velocity feedback control (i.e. $\delta_{1}=-0.1, \delta_{2}=0.02$ ). Generally, Fig. 5 illustrates that the stable solutions region is repeated periodically in $\tau_{1} \tau_{2}$-plane and its topology depends on the position and velocity gain values. Effect of increasing the velocity gain $\left(\delta_{2}\right)$ on the stability chart at $\delta_{1}=0.1$ and -0.1 is illustrated in Figs. 6 and 7, respectively. The figures show how the controller gains alter the stability chart topology, where the stable and unstable regions are longitudinal areas parallel to $\tau_{2}$-axis at $\delta_{1}>$ $\delta_{2}$, while at $\delta_{1}<\delta_{2}$ they become transversal regions parallel to $\tau_{1}$-axis. At $\delta_{1}=\delta_{2}$, unstable solutions regions become periodic and isolated areas as shown in Figs. 6b and 7b. Fig. 8 illustrates the controlled system frequencyresponse curves at different values of the time-delay $\left(\tau_{1}\right)$ that selected according to Fig. 5a when $\tau_{2}=\frac{\pi}{2}$. The figure shows the great influences of the time-delays on the controlled system FRC. It is noticed from the figure that the best conditions for vibration suppression occur at $\tau_{1}=\frac{3 \pi}{2}$ and $\tau_{2}=\frac{\pi}{2}$ (i.e. when the time-delays are corresponding to the center of stable solutions region of Fig. 5a). Influences of the time-delays selected according to Fig. 5 b (i.e. $\delta_{1}=-0.1, \delta_{2}=0.02$ ) on the controlled system FRC are illustrated in Fig. 9. The figure confirms that the best frequency response curve occurs at $\tau_{1}=\tau_{2}=\frac{\pi}{2}$ (i.e. when time-delays are selected within the center of stable solutions region shown in Fig. 5b). However, the existence of time-delays in the control loop can destabilize the system under control; it is possible enhancing the controller efficiency via selecting the loop-delays to be within the center of the stable solutions region. The system frequency-response curves before control and 
after applying the time-delayed position-velocity controller are compared analytically and numerically in Fig. 10 . By comparing Figs. 3 with 10, it is noticed that the time-delay improves the controller efficiency, effectively. Figs 11 and 12 illustrate the Jeffcott-rotor temporal vibrations before and after control according to the points shown on Fig. 10 that named a bistable solution and unique solution. The two figures were obtained at the system parameters $\sigma=0.035, \delta_{1}=\delta_{2}=\tau_{1}=\tau_{2}=0$ (without control), and $\sigma=0.035, \delta_{1}=0.1, \delta_{2}=0.02, \tau_{1}=$ $\frac{3 \pi}{2}, \tau_{2}=\frac{\pi}{2}$ (with time-delays control). Figs 11 and 12 were obtained via numerically integrating the system original equations (6) utilizing the DDE23 Matlab solver at the initial conditions $x_{1}=x_{2}=\dot{x}_{1}=\dot{x}_{2}=0$ and $x_{1}=1, x_{2}=-1, \dot{x}_{1}=\dot{x}_{2}=0$, respectively. The figures confirm the sensitivity of the system to the initial conditions before control, where the system has two different whirling-orbits before control, while these bistable motion has been diminished to small unique whirling-orbit after control. To investigate the Jeffcott-rotor lateral vibrations at a wide range of disc eccentricity $(f)$, the system eccentricity-response curve before control, after control, and after optimal time-delayed control is illustrated in Fig. 13 at $\sigma=0$. The figure demonstrates that involving time-delays in the control loop enhances the controller performance in suppressing the system lateral vibrations. Based on the above discussions and according to the obtained slow-flow modulating equations (17.117.4), the existence of time-delays in a control loop involving PD-controller make the equivalent linear damping coefficients of the controlled system function in the proportional gain, the derivative gain, and the loop-delays as mentioned after equations (17.1-17.4). Accordingly, for known loop-delays, selecting the controller gain so that maximize $\mu_{e 1}$ and $\mu_{e 2}$ will guarantee the best controller performance.

(a)

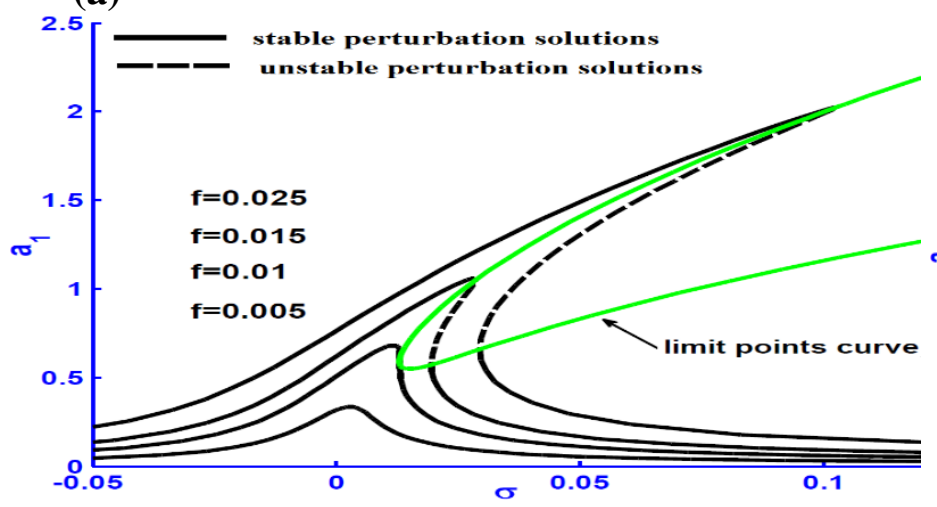

(b)

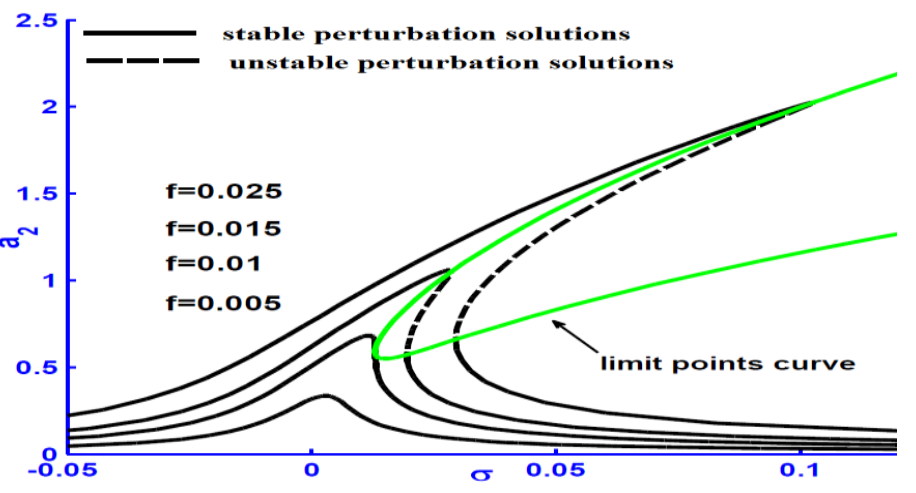

Figure 2. Uncontrolled system frequency-response curve at different values of the disc eccentricity $f$ : (a) $x_{1}$ mode, (b) $x_{2}$ mode.

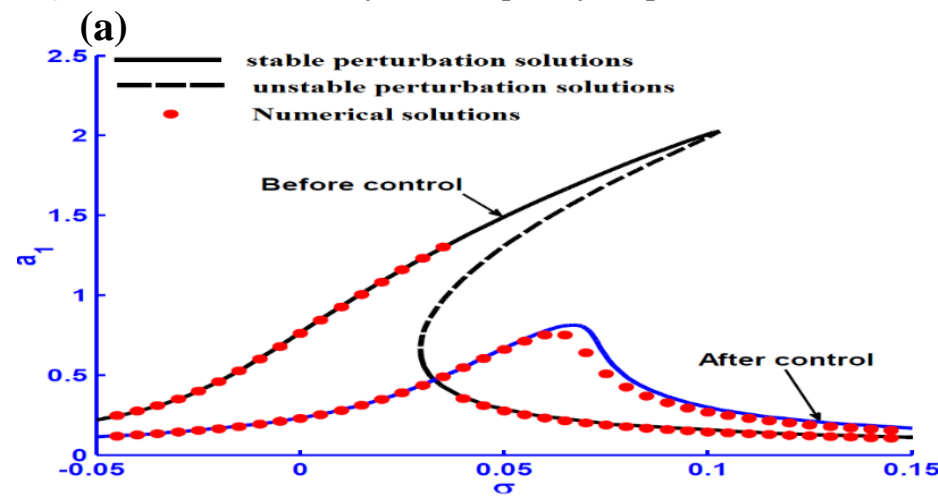

Figure 3. The system frequency-response curve before and after con (a)

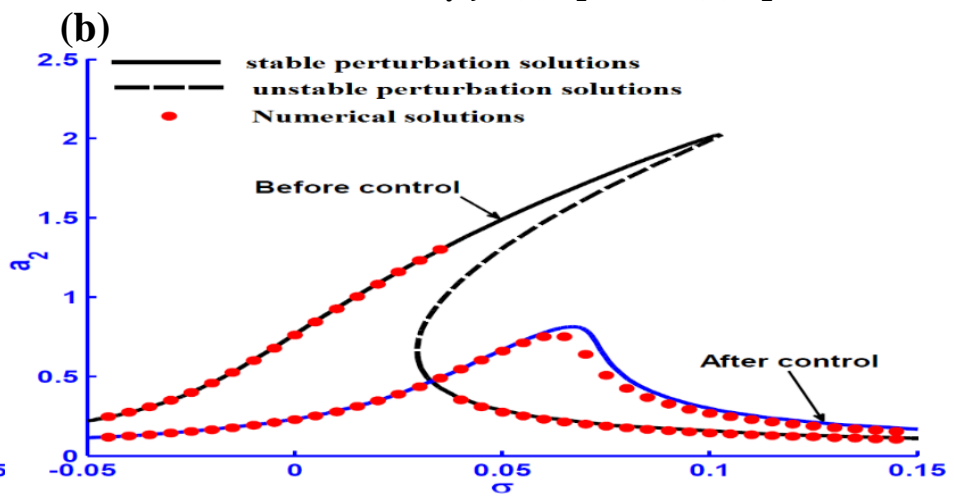



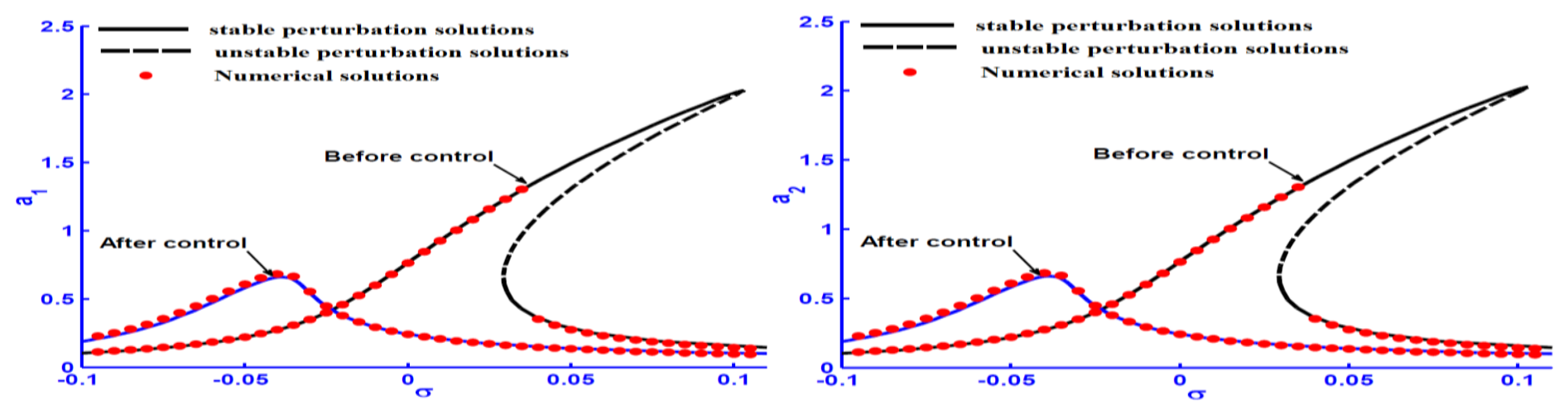

Figure 4. The system frequency-response curve before and after control at: $\delta_{1}=-0.1, \delta_{2}=0.02, \tau_{1}=\tau_{2}=0$, (a) $x_{1}$ mode, (b) $x_{2}$ mode

(a)

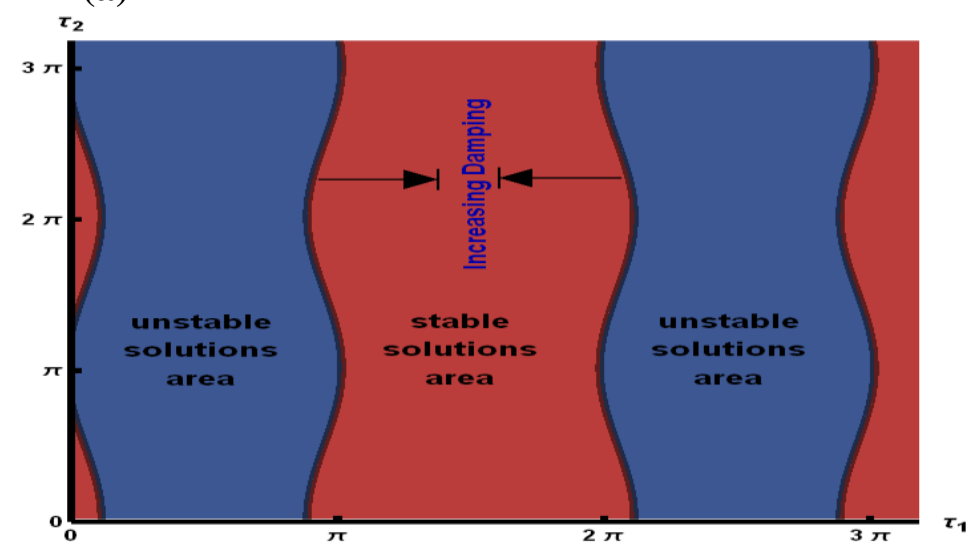

(b)

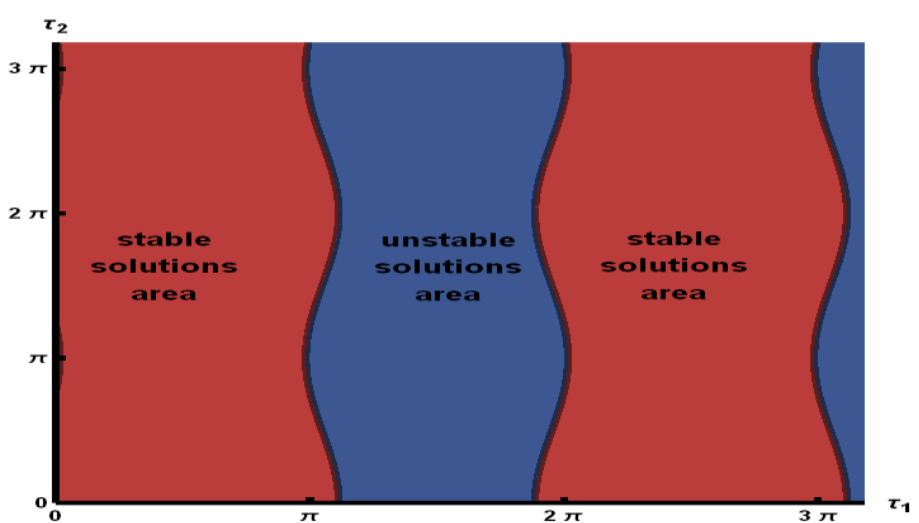

Figure 5. Modified linear damping coefficients $\mu_{\text {hori }}$ and $\mu_{\text {vert }}$ as functions of the time delays at control gains: (a) $\delta_{1}=0.1, \delta_{2}=0.02$, (b) $\delta_{1}=-0.1, \delta_{2}=0.02$

(a)

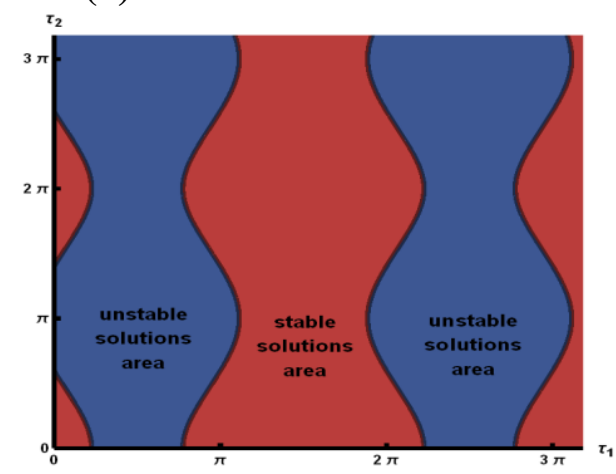

(b)

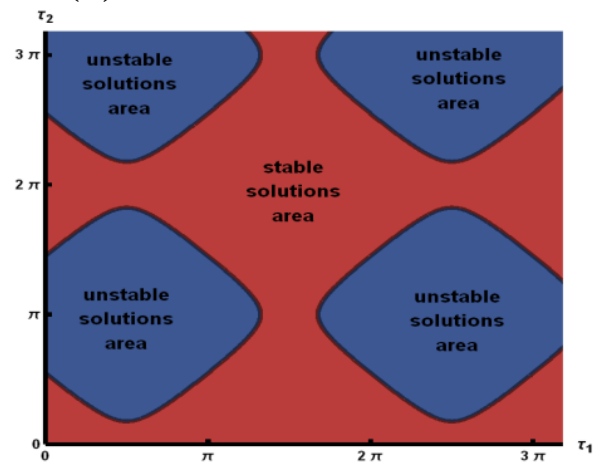

(c)

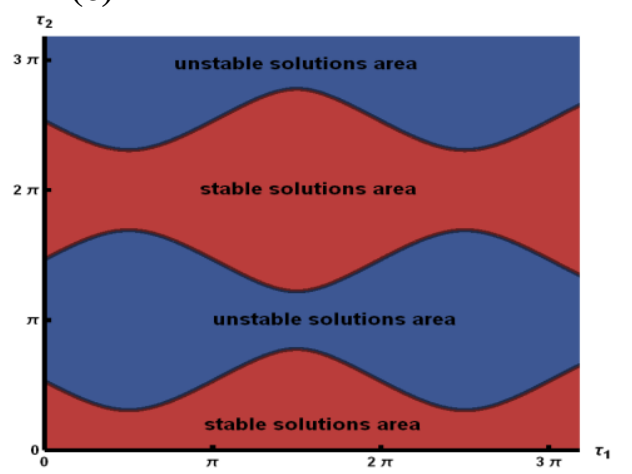

Figure 6. Modified linear damping coefficients $\mu_{\text {hori }}$ and $\mu_{\text {vert }}$ as functions of the time delays at control gains: (a) $\delta_{1}=0.1, \delta_{2}=0.05$, (b) $\delta_{1}=0.1, \delta_{2}=0.1$, and (c) $\delta_{1}=0.1, \delta_{2}=0.15$.

(a)

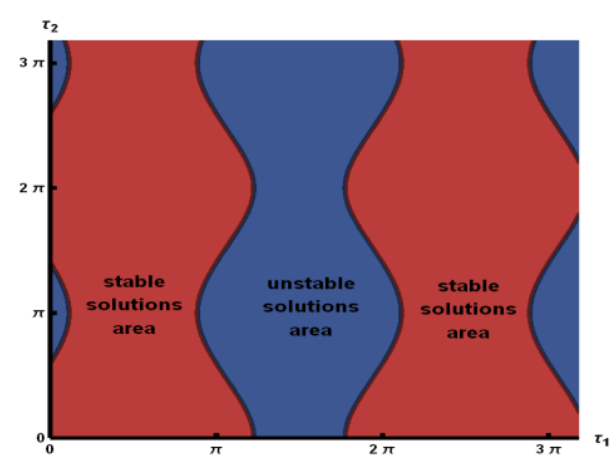

(b)

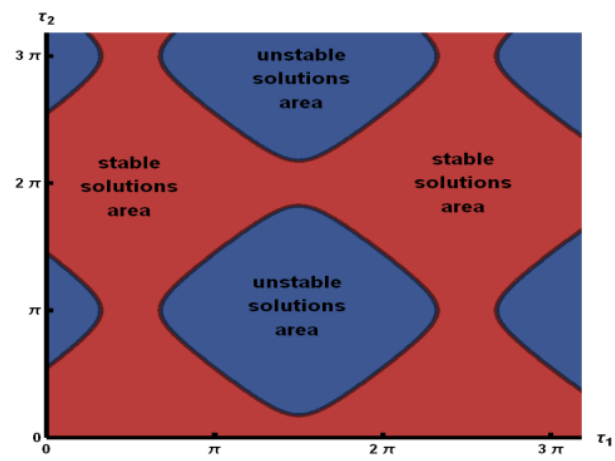

(c)

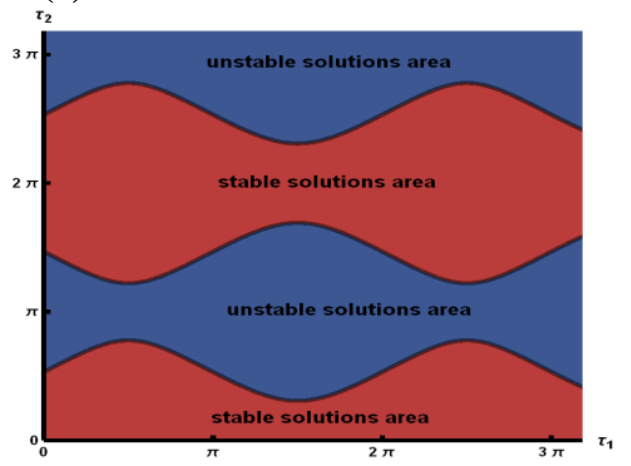


Figure 7. Modified linear damping coefficients $\mu_{\text {hori }}$ and $\mu_{\text {vert }}$ as functions of the time delays at control gains: (a) $\delta_{1}=-0.1, \delta_{2}=$ 0.05 , (b) $\delta_{1}=-0.1, \delta_{2}=0.1$, and (c) $\delta_{1}=-0.1, \delta_{2}=0.15$.

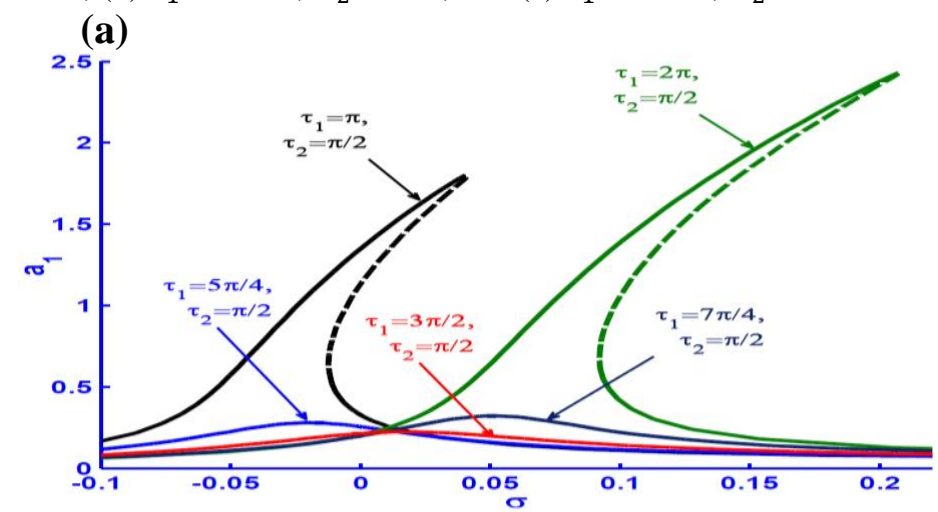

(b)

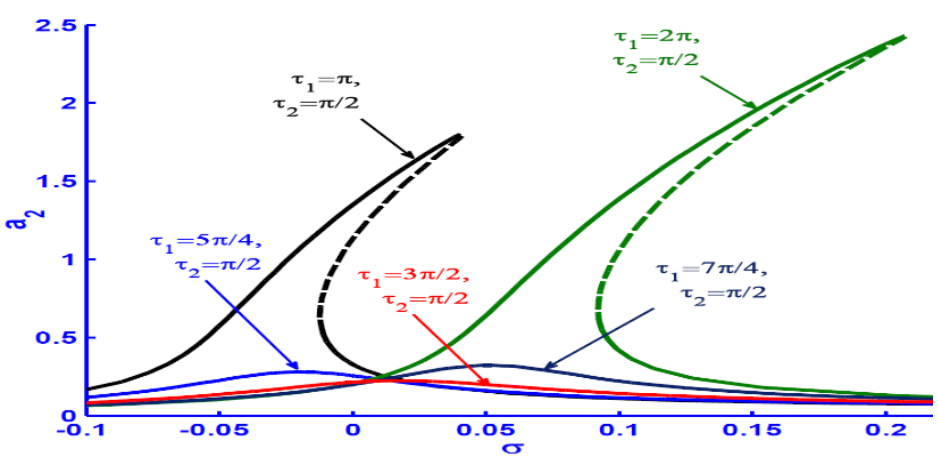

Figure 8. The controlled system frequency-response curve when $\delta_{1}=0.1, \delta_{2}=0.02$ at different values of the time delays $\tau_{1}, \tau_{2}$ : (a) $x_{1}$ mode, (b) $x_{2}$ mode

(a)

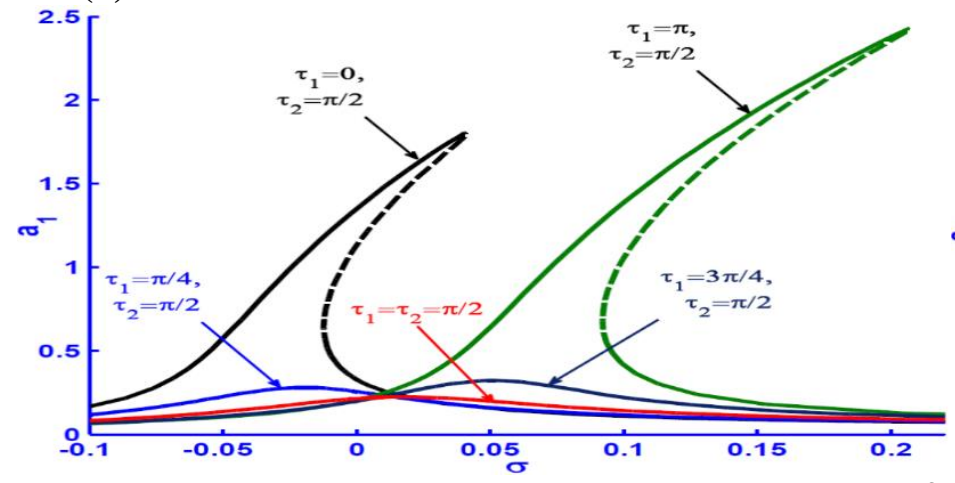

(b)

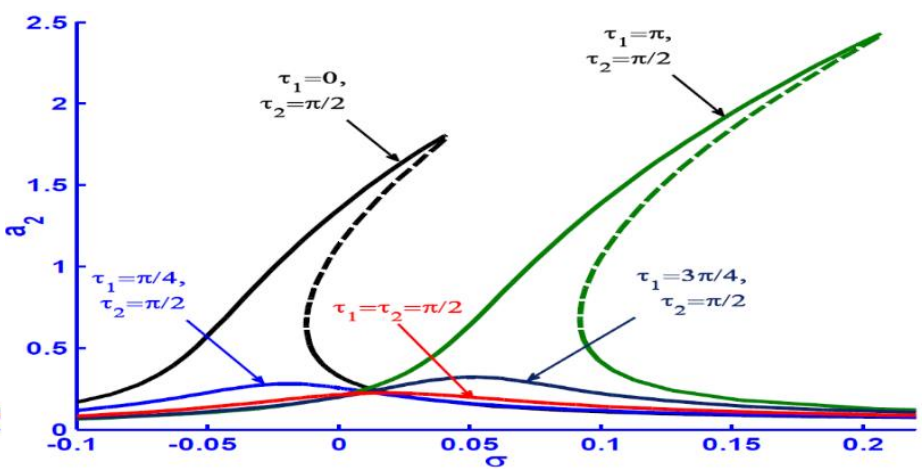

Figure 9. The controlled system frequency-response curve when $\delta_{1}=-0.1, \delta_{2}=0.02$ at different values of the time delays $\tau_{1}, \tau_{2}$ : (a) $x_{1}$ mode, (b) $x_{2}$ mode

(a)

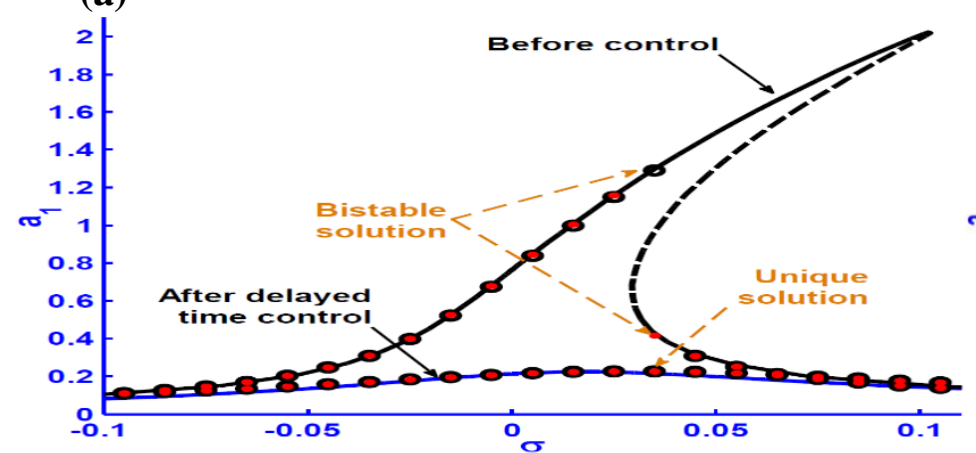

(b)

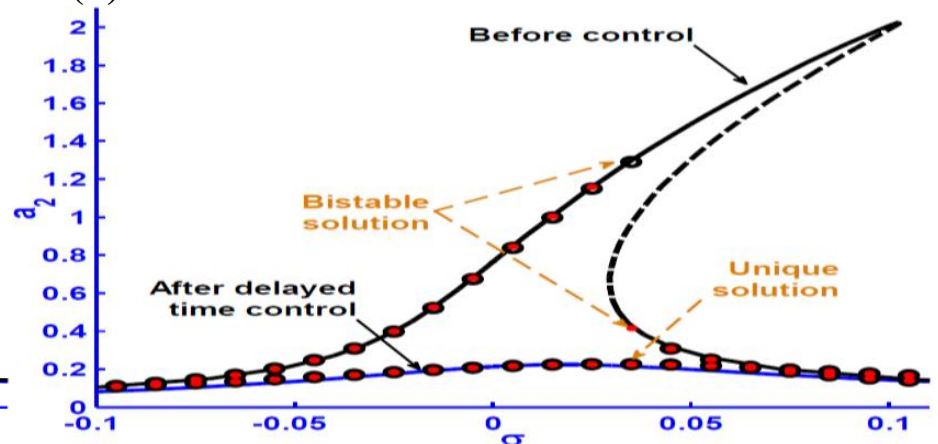

Figure 10. System frequency-response curve before control $\left(\delta_{1}=\delta_{2}=0\right)$, and after control $\left(\delta_{1}=0.1, \delta_{2}=0.02\right)$ with the optimal delayed times $\left(\tau_{1}=\frac{3 \pi}{2}, \tau_{2}=\frac{\pi}{2}\right)$ : (a) $x_{1}$ mode, (b) $x_{2}$ mode

(a)

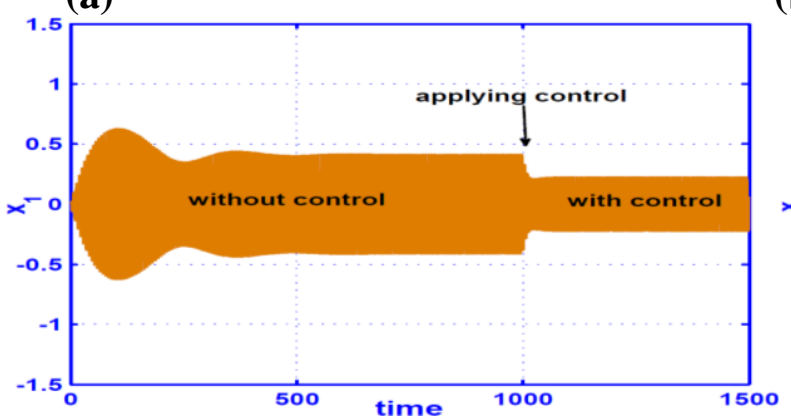

(b)

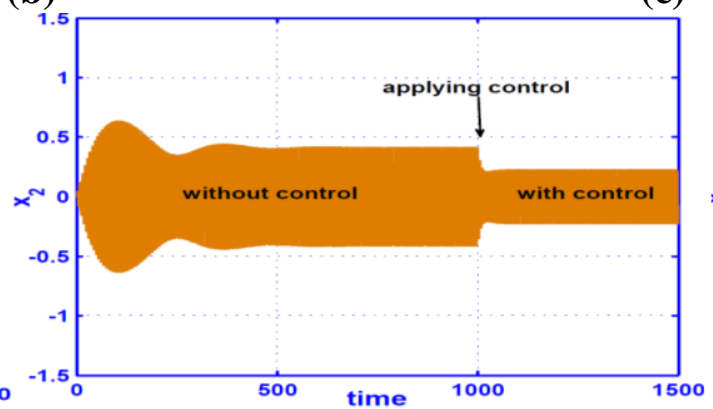

(c)

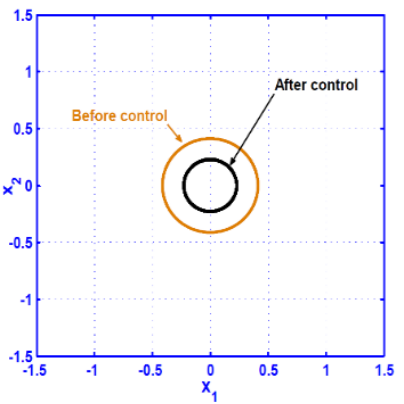

Figure 11. (a, b) time histories, and (c) long-time orbit plot at: $\sigma=0.035, \delta_{1}=0.1, \delta_{2}=0.02, \tau_{1}=\frac{3 \pi}{2}, \tau_{2}=\frac{\pi}{2}$ and initial conditions $x_{1}=x_{2}=\dot{x}_{1}=\dot{x}_{2}=0$ 
(a)

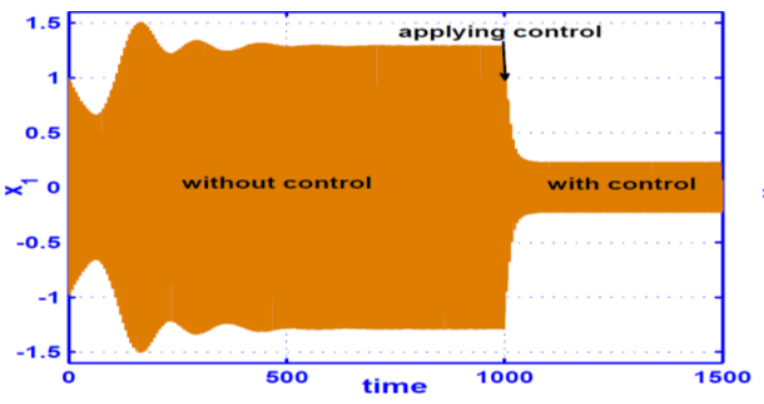

(b)

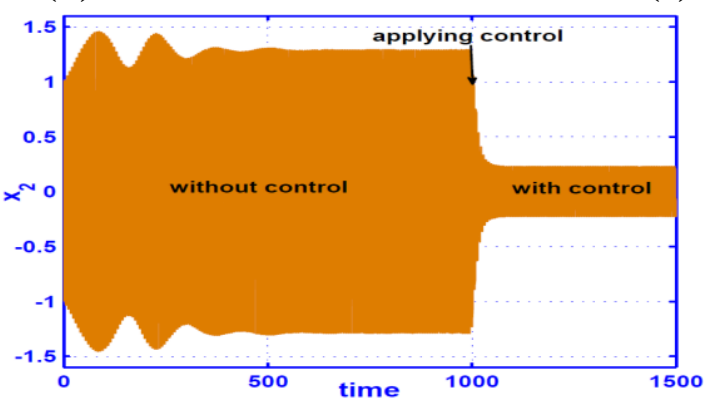

(c)

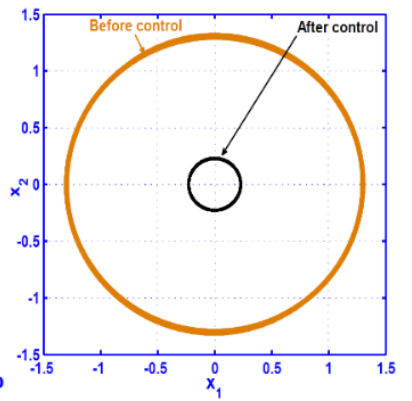

Figure 12. (a, b) time histories, and (c) long-time orbit plot at: $\sigma=0.035, \delta_{1}=0.1, \delta_{2}=0.02, \tau_{1}=\frac{3 \pi}{2}, \tau_{2}=\frac{\pi}{2}$ and initial conditions $x_{1}=1, x_{2}=-1, \dot{x}_{1}=\dot{x}_{2}=0$

(a)

(b)
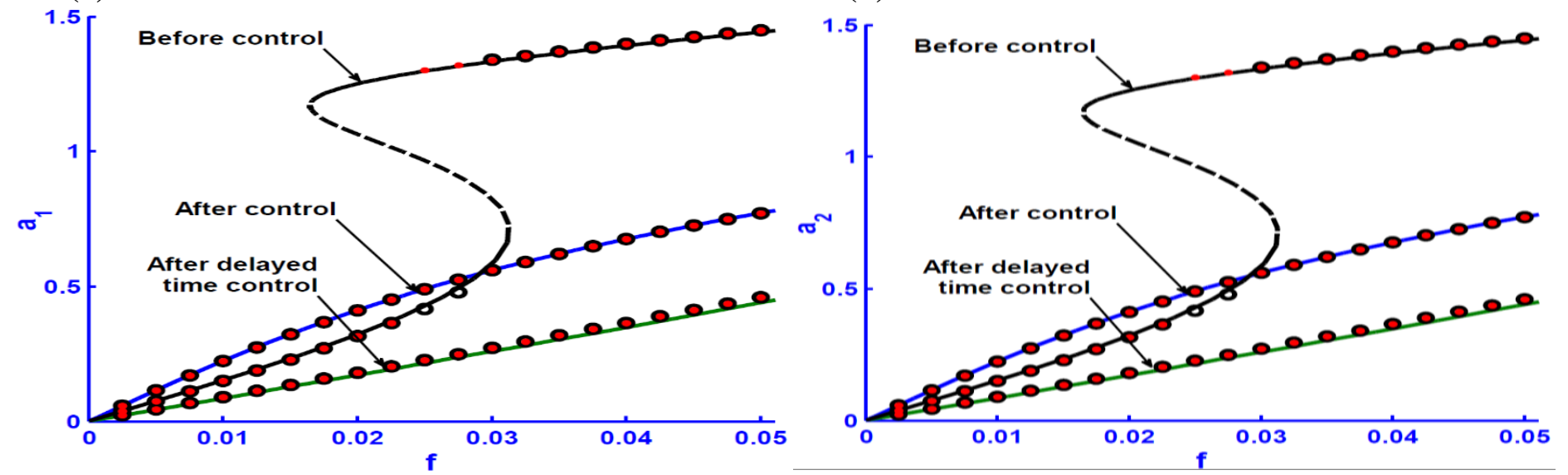

Figure 13. The controlled system eccentricity-response curve without control $\left(\delta_{1}=\delta_{2}=0\right)$, with control $\left(\delta_{1}=0.1, \delta_{2}=0.02\right)$, and with delayed-time control $\left(\delta_{1}=0.1, \delta_{2}=0.02, \tau_{1}=\frac{3 \pi}{2}, \tau_{2}=\frac{\pi}{2}\right)$ at $\sigma=0.035$ : (a) $x_{1}$ mode, (b) $x_{2}$ mode

\section{Concluded remarks}

Within this work, time-delayed position-velocity controller is proposed to control the nonlinear whirling motions of a vertically suspended Jeffcott-rotor system. Multiple time scales perturbation method is utilized to obtain the slow-flow modulating equations that governing the whole system dynamics. The obtained equations are employed to investigate the effects of both the loop-delays and control gain on the system response curves. Based on the above discussions we may conclude the following:

1. The stable solutions area in $\tau_{1} \tau_{2}$ - plane is repeated periodically, where its topology depends on the controller gains.

2. However the time-delay in the closed loop control system is one of the main reasons of the loop instability; it is possible to improve the position-velocity feedback controller efficiency via selecting the time-delays that maximize $\mu_{e 1}$ and $\mu_{e 2}$.

3. At specific values of the loop-delays, the position-controller acts as a velocity-controller and vice versa.

4. The center of the stable-solutions area shown in $\tau_{1} \tau_{2}$-plane represents the optimal values of loop-delays for vibration suppression.

5. At small unintentional loop-delays, the integration of positive-position and negative-velocity feedback controller to the targeted system is the optimal gain selection for enhancing the controller efficiency.

\section{References}

[1] H. Hu, E. H. Dowell, L. N. Virgin, Resonances of a harmonically forced Duffing oscillator with time delay state feedback, Nonlinear Dyn, 15, (1998), 311-327

[2] A. Maccari, The response of a parametrically excited van der pol oscillator to a time delay state feedback, Nonlinear Dyn, 26, (2001),105-119

[3] A. Maccari, Vibration control for the primary resonance of the van der Pol oscillator by a time delay state feedback, International Journal of Non-Linear Mechanics, 38, (2003), 123-131 
[4] A. Maccari,Vibration control for the primary resonance of a cantilever beam by a time delay state feedback, JSV,259(2), (2003), 241-251

[5] A. Maccari, Vibration amplitude control for a van der Pol-Duffing oscillator with time delay, JSV, 317, (2008), 20-29

[6] A. Maccari, Arbitrary amplitude periodic solutions for parametrically excited systems with time delay, Nonlinear Dyn,51, (2008),111-126

[7] T. M. Morrison, R. H. Rand, 2:1 Resonance in the delayed nonlinear Mathieu equation, Nonlinear Dyn, 50, (2007), 341-352

[8] S. M., Sah, R. H. Rand, Using delay to quench undesirable vibrations, Nonlinear Dyn, 62, (2010), 407-416

[9] S. Shao, K. M. Masri, M. I. Younis, The effect of time-delayed feedback controller on an electrically actuated resonator, Nonlinear Dyn, 74, (2013), 257-270

[10] A. M. Elnaggar, K. M. Khalil, The response of nonlinear controlled system under an external excitation via time delay state feedback, Journal of King Saud University - Engineering Sciences, 28, (2016),75-83

[11] K. A. Alhazza, M. F. Daqaq, A. H. Nayfeh, D. J. Inman, Nonlinear vibrations of parametrically excited cantilever beams subjected to non-linear delayed-feedback control, International Journal of Non-Linear Mechanics, 43, (2008), $801-812$

[12] K. A. Alhazza, M. A. Majeed, Free vibrations control of a cantilever beam using combined time delay feedback, JVC,18(5), (2011), 609-621

[13] M. F. Daqaq, K. A. Alhazza, Y. Qaroush, On primary resonances of weakly nonlinear delay systems with cubic nonlinearities, Nonlinear Dyn, 64, (2011), 253-277

[14] Ling Xu, Lei Chen, Weili Xiong, Parameter estimation and controller design for dynamic systems from the step responses based on the Newton, Nonlinear Dyn, 79, (2015), 2155-2163

[15] Ling $\mathrm{Xu}, \mathrm{A}$ proportional differential control method for a time-delay system using the Taylor expansion approximation, Applied Mathematics and Computation 236, (2014, 391-399

[16] N. A. Saeed, W. A. El-Ganaini, Time-delayed control to suppress the nonlinear vibrations of a horizontally suspended Jeffcott-rotor system, Applied Mathematical Modelling, 44, (2017), 523-539

[17] N. A. Saeed, W. A. El-Ganaini, Utilizing time-delays to quench the nonlinear vibrations of a two-degree-offreedom system, Meccanica, doi: 10.1007/s11012-017-0643-z

[18] Y. Ishida, T. Inoue, Vibration Suppression of nonlinear rotor systems using a dynamic damper, JVC, 138, (2007),1127-1143

[19] A. Nayfeh, D. Mook, Nonlinear Oscillations. Wiley, New York (1995).

[20] L.F. Shampine, S. Thompson, Solving DDEs in MATLAB. Applied Numerical Mathematics, 37, (2001), $441-458$ 